\title{
High-energy gamma- and cosmic-ray observations with future space-based GAMMA-400 gamma-ray telescope
}

\author{
N.P. Topchiev ${ }^{1, *}$, A.M. Galper ${ }^{1,2}$, I.V. Arkhangelskaja ${ }^{2}$, A.I. Arkhangelskiy ${ }^{1,2}$, A.V. Bakaldin ${ }^{1,3}$, I.V. Chernysheva ${ }^{1,2}$, \\ O.D. Dalkarov ${ }^{1}$, A.E. Egorov ${ }^{1}$, Yu.V. Gusakov ${ }^{1}$, M.D. Kheymits ${ }^{2}$, A.A. Leonov ${ }^{1,2}$, P.Yu. Naumov ${ }^{2}$, N.Yu. Pappe ${ }^{1}$, M.F. \\ Runtso $^{2}$, Yu.I. Stozhkov ${ }^{1}$, S.I. Suchkov ${ }^{1}$, Yu.T. Yurkin ${ }^{2}$, and V.G. Zverev ${ }^{1}$ \\ ${ }^{1}$ Lebedev Physical Institute, RU-119991 Moscow, Russia \\ ${ }^{2}$ National Research Nuclear University MEPhI (Moscow Engineering Physics Institute), RU-115409 Moscow, Russia \\ ${ }^{3}$ Scientific Research Institute for System Analysis, RU-117218 Moscow, Russia

\begin{abstract}
The future space-based GAMMA-400 gamma-ray telescope will be installed on the Navigator platform of the Russian Astrophysical Observatory. A highly elliptical orbit will provide observations for 7-10 years of many regions of the celestial sphere continuously for a long time ( 100 days). GAMMA-400 will measure gamma-ray fluxes in the energy range from $\sim 20 \mathrm{MeV}$ to several $\mathrm{TeV}$ and electron + positron fluxes up to $\sim 20 \mathrm{TeV}$. GAMMA-400 will have an excellent separation of gamma rays from the background of cosmic rays and electrons + positrons from protons and an unprecedented angular $\left(\sim 0.01^{\circ}\right.$ at $\left.\mathrm{E}_{\gamma}=100 \mathrm{GeV}\right)$ and energy $\left(\sim 1 \%\right.$ at $\left.\mathrm{E}_{\gamma}=100 \mathrm{GeV}\right)$ resolutions better than for Fermi-LAT, as well as ground-based facilities, by a factor of 5-10. Observations of GAMMA-400 will provide new fundamental data on discrete sources and spectra of gamma-ray emission and electrons + positrons, as well as the nature of dark matter.
\end{abstract}

\section{Introduction}

At present AGILE, Fermi-LAT, CALET, DAMPE perform observations of discrete gamma-ray sources in space. The third Fermi-LAT catalog (3FGL) contains 3033 sources for the energy range from $100 \mathrm{MeV}$ to $300 \mathrm{GeV}$, but $33 \%$ of gamma-ray sources are unidentified [1]. The groundbased facilities VERITAS, MAGIC, H.E.S.S., HAWC and others observe only 215 gamma-ray sources in the energy range above $100 \mathrm{GeV}$ (http://tevcat.uchicago.edu/). It is important to note that the observational data were mainly obtained for the energy ranges $<100 \mathrm{GeV}$ for Fermi-LAT and $>100 \mathrm{GeV}$ for ground-based facilities and these data overlap poorly for many gamma-ray sources. The frontier range around $100 \mathrm{GeV}$ is still very interesting for investigation.

Another very interesting and important goal in the studies of gamma-ray sky is indirect searches of dark matter (DM). WIMPs with mass between several $\mathrm{GeV}$ and several $\mathrm{TeV}$ are still considered as the most probable candidate. WIMPs can annihilate or decay with the production of gamma rays. This emission can have both continuous energy spectrum or mono-energetic narrow lines. Up to now, there are no data on DM gamma-ray lines from space- and ground-based instruments.

Fermi-LAT, PAMELA, AMS-2, CALET, DAMPE obtained energy spectra for primarily cosmic-ray (CR) electrons + positrons, but their fluxes practically do not match in the energy range more than $50 \mathrm{GeV}$ [2].

\footnotetext{
*Corresponding author e-mail: tnp51@yandex.ru
}

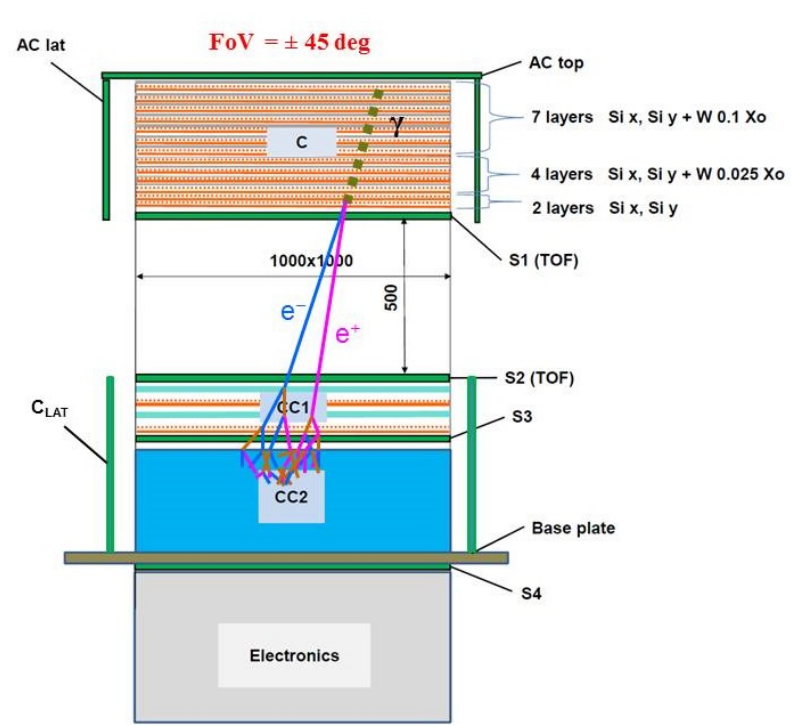

Figure 1. The GAMMA-400 physical scheme

Therefore new direct observations of gamma-ray emission in the energy range from $\sim 20 \mathrm{MeV}$ up to several $\mathrm{TeV}$ and electron + positron fluxes up to $20 \mathrm{TeV}$ are required using the space-based telescope with much better separation from background, angular and energy resolutions in order to identify many gamma-ray sources, resolve DM gamma-ray lines and clarify energy spectra of CR electrons + positrons. 


\section{THE GAMMA-400 gamma-ray telescope}

The GAMMA-400 gamma-ray telescope will be installed onboard the Russian space astrophysical observatory [35]. The GAMMA-400 main scientific goals are: precise uninterrupted up to hundred days measurements of Galactic Center, Fermi Bubbles, Crab, Vela, Cygnus, Geminga, Sun, and other regions, extended and point gamma-ray sources, diffuse gamma rays, dark matter searching, measuring electron + positron fluxes with unprecedented an$\operatorname{gular}\left(\sim 0.01^{\circ}\right.$ at $\left.\mathrm{E}_{\gamma}=100 \mathrm{GeV}\right)$ and energy $(\sim 1 \%$ at $\left.\mathrm{E}_{\gamma}=100 \mathrm{GeV}\right)$ resolutions.

\subsection{The GAMMA-400 physical scheme}

The physical scheme of the GAMMA-400 gamma-ray telescope is shown in Fig. 1.

GAMMA-400 includes:

- plastic scintillation anticoincidence top ACtop $(1280 \times 1280 \mathrm{~mm})$ and four lateral AClat $(1280 \times 600$ $\mathrm{mm}$ ) detectors with efficiency of 0.99995 and time resolution of $300 \mathrm{ps}$;

- converter-tracker (C), consisting of 13 pairs of planes of silicon strip detectors ( $\mathrm{X}$ and Y coordinates, $1000 \times 1000$ $\mathrm{mm}$ ) with pitch of $0.08 \mathrm{~mm}$ and analog readout. First 7 and 4 pairs have $\mathrm{W}$ converter foils of $0.1 \mathrm{X}_{0}$ and 0.025 $\mathrm{X}_{0}$ thick each, respectively. Last 2 pairs have no $\mathrm{W}$;

- time of flight system (ToF) consisting of plastic scintillation detectors (S1 and S2, 1000×1000 mm) spaced by $500 \mathrm{~mm}$ with coefficient of separation for top/down moving particles of 1000 and time resolution of 300 ps;

- calorimeter (CC1 and CC2). CC1 (1000×1000 mm) consists of two layers of $\mathrm{CsI}(\mathrm{Tl})$ scintillation crystals and silicon strip detectors ( $\mathrm{X}$ and $\mathrm{Y}$ coordinates) with pitch of $0.08 \mathrm{~mm}$. CC2 $(1000 \times 1000 \mathrm{~mm})$ consists of $28 \times 28 \mathrm{CsI}(\mathrm{Tl})$ crystals. Total thickness of calorimeter is $\sim 22 \mathrm{X}_{0}\left(\sim 1.0 \lambda_{0}\right)$ and $\sim 54 \mathrm{X}_{0}\left(\sim 2.5 \lambda_{0}\right)$ for vertical and lateral particle detection, respectively;

- plastic scintillation detectors (S3 and S4, 1000×1000 $\mathrm{mm}$ ) for improving hadronic and electromagnetic shower separation;

- four lateral scintillation detectors of calorimeter (Clat) for detecting lateral particles.

After interaction of incident gamma rays with the GAMMA-400 matter the backscattering omnidirectional particles (mainly 1-MeV photons and electrons) are arisen. Figure 2 shows the simulation result for primary $100-\mathrm{GeV}$ gamma ray. In order to exclude backscattering particles, all scintillation detectors consist of two independent 10$\mathrm{mm}$ layers and fast timing methods are used.

The GAMMA-400 energy range for gamma-ray studies is from $\sim 20 \mathrm{MeV}$ to several $1 \mathrm{TeV}$ and up to $\sim 20 \mathrm{TeV}$ for electrons + positrons. The field of view (FoV) for detecting particles from top is $\pm 45^{\circ}$. The geometrical factor for detecting electrons + positrons from vertical and four lateral directions is $\sim 3 \mathrm{~m}^{2} \mathrm{sr}$.

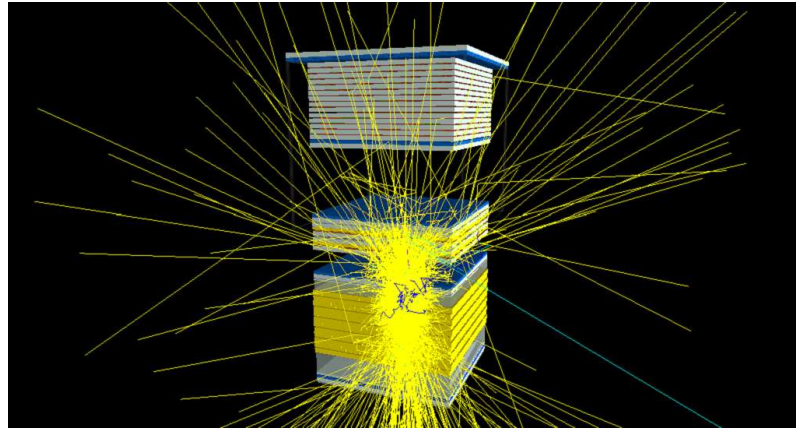

Figure 2. The interaction of the $100-\mathrm{GeV}$ gamma ray with the GAMMA-400 matter with the formation of backscattering particles. Secondary gammas, positrons and electrons are marked by yellow, violet and blue colors respectively

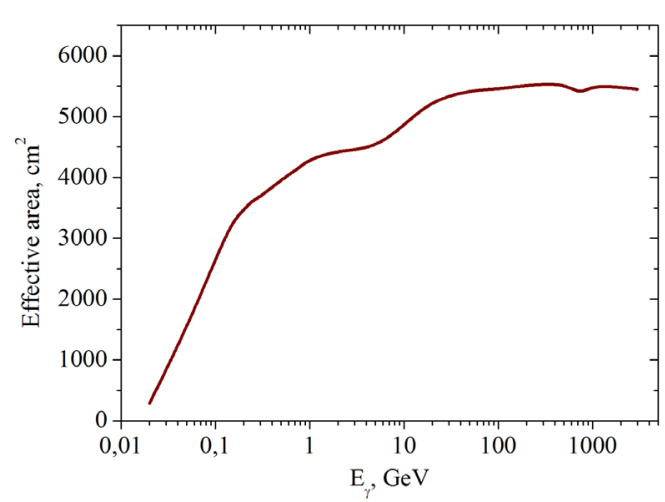

Figure 3. The dependence of the effective area vs the energy for vertically incident particles

\subsection{The GAMMA-400 performance}

Model calculations of the GAMMA-400 gamma-ray telescope performance were carried out using the "GEANT4.10.01.p02" software package. As a result of calculations, we obtained the following dependences:

- the effective area vs the energy (Fig. 3), which is $\sim 5000$ $\mathrm{cm}^{2}$ for energies greater than $10 \mathrm{GeV}$;

- the effective area vs the angle of incidence of particles for $\mathrm{E}_{\gamma}=1,10,100 \mathrm{GeV}$ (Fig. 4);

- the energy resolution vs the energy (Fig. 5). The energy resolution for $\mathrm{E}_{\gamma}=100 \mathrm{GeV}$ is $\sim 1 \%$;

- the angular resolution vs the energy (Fig. 6). The angular resolution for $\mathrm{E}_{\gamma}=100 \mathrm{GeV}$ is $\sim 0.01^{\circ}$.

Using the combined information from all GAMMA400 detector systems, it is possible to reach an effective rejection of protons from electrons. The methods to separate electron from protons presented in [6] are based on the difference of the development of hadronic and electromagnetic showers inside the instrument. For the current physical scheme the rejection factor for vertical protons is about $3 \times 10^{5}$. 


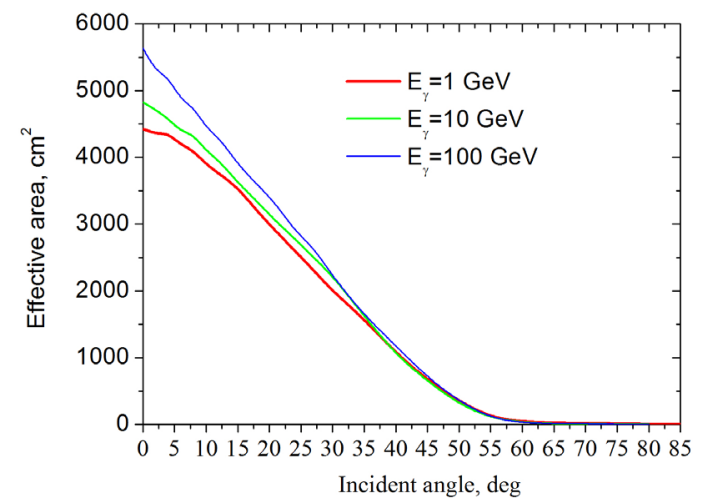

Figure 4. The dependence of the effective area vs the angle of incidence of particles for $\mathrm{E}_{\gamma}=1 \mathrm{GeV} ; \mathrm{E}_{\gamma}=10 \mathrm{GeV} ; \mathrm{E}_{\gamma}=100$ $\mathrm{GeV}$

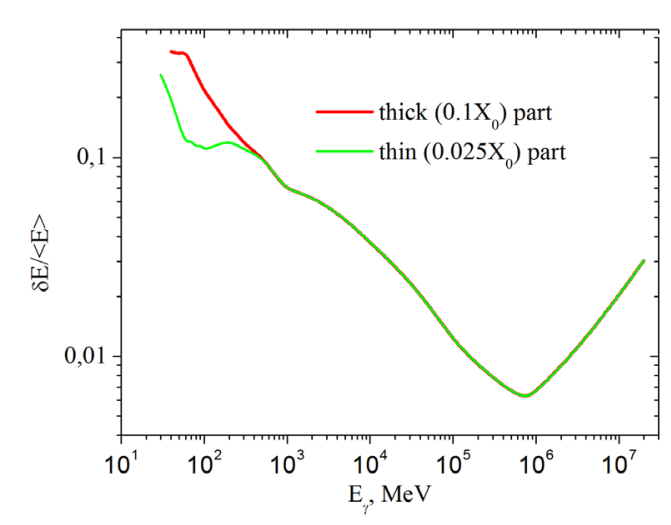

Figure 5. The dependence of the energy resolution vs the energy for two parts of the converter: 4 panels with $\mathrm{W}=0.025 \mathrm{X}_{0}$ and 7 panels with $\mathrm{W}=0.1 \mathrm{X}_{0}$

\subsection{The GAMMA-400 astrophysical observatory}

The GAMMA-400 astrophysical observatory will be installed onboard of the Navigator space platform, which is designed and manufactured by the Lavochkin Association and includes a gamma-ray telescope, an X-ray telescope and plasma detectors.

Using the Navigator space platform gives the GAMMA-400 experiment a highly unique opportunity for the near future gamma- and cosmic-ray science, since it allows us to install a scientific payload (mass of $\sim 4100 \mathrm{~kg}$, power consumption of $2000 \mathrm{~W}$, and telemetry downlink of $100 \mathrm{~GB} /$ day, with lifetime more than 7 years), which will provide GAMMA-400 by the means to significantly contribute as the next generation instrument for gamma-ray astronomy and cosmic-ray physics.

The GAMMA-400 experiment will be initially launched into a highly elliptical orbit (with an apogee of $300000 \mathrm{~km}$ and a perigee of $500 \mathrm{~km}$, with an inclination of $51.4^{\circ}$ ), with 7 days orbital period. Under the influence of gravitational disturbances of the Sun, Moon and the Earth after $\sim 6$ months the orbit will transform to about an ap-

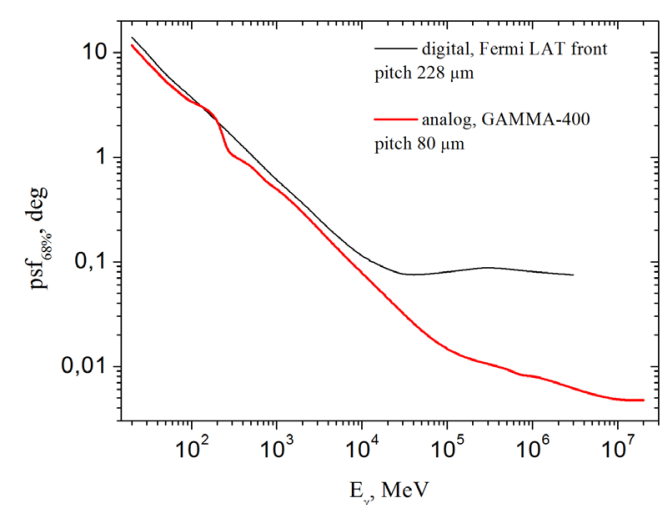

Figure 6. The dependence of the angular resolution vs the energy for: GAMMA-400 (80 $\mu \mathrm{m}$ pitch, analog readout) and Fermi-LAT (228 $\mu \mathrm{m}$ pitch, digital readout)

proximately circular one with a radius of $\sim 200000 \mathrm{~km}$ and will not suffer from the Earth's occultation and be outside the radiation belts. A great advantage of such an orbit is the fact that the full sky coverage will always be available for gamma-ray astronomy, since the Earth will not cover a significant fraction of the sky, as is usually the case for low-Earth orbit. Therefore, the GAMMA-400 source pointing strategy will hence be properly defined to maximize the physics outcome of the experiment. The launch of the GAMMA-400 space observatory is planned for the middle of the 2020 s.

\subsection{Comparison of GAMMA-400 with Fermi-LAT and ground-based facilities}

The GAMMA-400 gamma-ray telescope has numerous advantages in comparison with the Fermi-LAT:

- highly elliptical orbit (without the Earth's occultation and away from the radiation belts) allows us to continuously observe with an aperture of $\pm 45^{\circ}$ different gamma-ray sources over a long period of time with the exposition greater by a factor of 8 than for Fermi-LAT operating in the sky-survey mode;

- thanks to a smaller pitch (by a factor of 3) and ana$\log$ readout in the coordinate silicon strip detectors, GAMMA-400 has an excellent angular resolution;

- due to the deep $\left(\sim 22 \mathrm{X}_{0}\right)$ calorimeter, GAMMA-400 has an excellent energy resolution and can provide more reliably the detection of gamma rays up to several $\mathrm{TeV}$ for vertically incident events;

- owing to the better gamma-ray separation from cosmic rays (in contrast to Fermi-LAT, the presence of a special trigger with event timing, time-of-flight system, twolayer scintillation detectors), GAMMA-400 is significantly well equipped to separate gamma rays from the background of cosmic rays and backscattering events.

GAMMA-400 will also have better angular and energy resolutions in the energy region $10-1000 \mathrm{GeV}$ in comparison with current and future space- and ground-based 


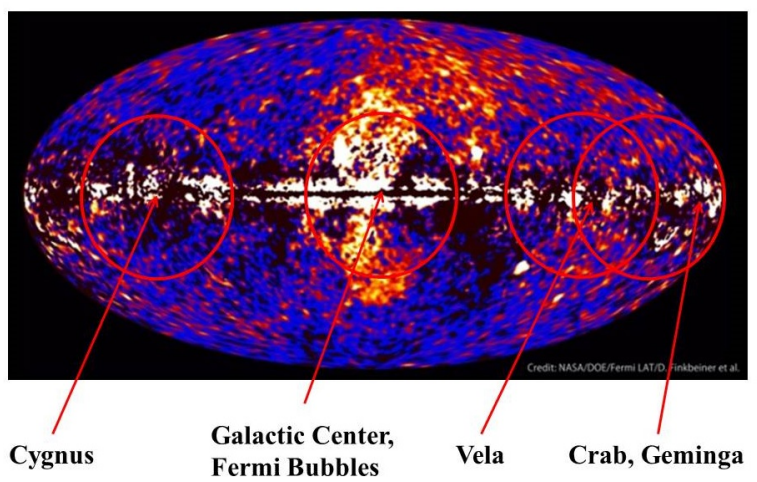

Figure 7. Galactic center, Fermi Bubbles, Crab, Cygnus, Vela, geminga and other regions will be observed with the GAMMA$400 \mathrm{FoV}$ of $\pm 45^{0}$

Table 1. Expected number of sources, $\mathrm{N}_{s}$, and gammas, $\mathrm{N}_{\gamma}$, for different energy ranges, when GAMMA-400 will observe several regions during 100 days

\begin{tabular}{|l|c|c|c|c|c|c|}
\hline $\begin{array}{l}\text { Energy } \\
\text { range }\end{array}$ & \multicolumn{2}{|c|}{$100 \mathrm{MeV}-$} & \multicolumn{2}{c|}{$1 \mathrm{GeV}-$} & \multicolumn{2}{c|}{$10 \mathrm{GeV}-$} \\
& \multicolumn{2}{|c|}{$100 \mathrm{GeV}$} & \multicolumn{2}{c|}{$100 \mathrm{GeV}$} & \multicolumn{2}{c|}{$100 \mathrm{GeV}$} \\
\hline Direction & $\mathrm{N}_{s}$ & $\mathrm{~N}_{\gamma}$ & $\mathrm{N}_{s}$ & $\mathrm{~N}_{\gamma}$ & $\mathrm{N}_{s}$ & $\mathrm{~N}_{\gamma}$ \\
\hline $\begin{array}{l}\text { Galactic } \\
\text { center } \\
\mathrm{b}=0^{0}, \\
\mathrm{l}=0^{0}\end{array}$ & 723 & $5.2 \times 10^{5}$ & 422 & $4.8 \times 10^{4}$ & 21 & 1365 \\
\hline $\begin{array}{l}\text { Crab } \\
\text { Geminga } \\
\mathrm{b}=0^{0}, \\
\mathrm{l}=190^{0}\end{array}$ & 495 & $3.1 \times 10^{5}$ & 175 & $3.9 \times 10^{4}$ & 11 & 1020 \\
\hline $\begin{array}{l}\text { Vela } \\
\mathrm{b}=0^{0}, \\
\mathrm{l}=265^{0}\end{array}$ & 649 & $5.2 \times 10^{5}$ & 280 & $6.3 \times 10^{4}$ & 9 & 1165 \\
\hline $\begin{array}{l}\text { Cygnus } \\
\mathrm{b}=0^{0}, \\
\mathrm{l}=75^{0}\end{array}$ & 604 & $3.2 \times 10^{5}$ & 269 & $3.1 \times 10^{4}$ & 12 & 1010 \\
\hline
\end{tabular}

instruments: VERITAS, MAGIC, H.E.S.S., CTA, and HAWC and it allows us to fill the data gap at an energy of $\sim 100 \mathrm{GeV}$ between the space- and ground-based instruments.

\section{The preliminary GAMMA-400 scientific program}

\subsection{Galactic plane}

GAMMA-400 will study continuously over a long period of time different regions of Galatic plane (Fig. 7), for example, Galactic center, Fermi Bubbles, Crab, Vela, Cygnus, Geminga with FoV of $\pm 45^{\circ}$. Using the gammaray fluxes from $3 \mathrm{FGL}$, we can expect the number of sources $\left(\mathrm{N}_{s}\right)$ and gammas $\left(\mathrm{N}_{\gamma}\right)$ for different energy ranges, when GAMMA-400 will observe several regions during 100 days (Table1).

\subsection{Dark matter searching}

Main targets to search for gamma rays from dark matter are:

Milky Way. The center of Milky Way is, apparently, the best potential source of dark matter emission possessing the largest J-factor [7]. Moreover, recently, the anomaly excess of gamma-ray emission in the GeV energy range was revealed near the Galactic center (the region of about one degree) [8], which can be well described by dark matter with mass of several tens of $\mathrm{GeV}$ and annihilation cross section of about standard thermal $10-26 \mathrm{~cm}^{3} / \mathrm{s}$. However, this observed excess can have another interpretation - the presence of a population of millisecond pulsars [9]. Therefore, the new GAMMA-400 observational data can help to solve this problem.

Milky Way satellites are considered for a long time as the strongest sources of constraints for dark matter, because they have sufficiently large J-factors and at the same time have considerably less gamma-ray background in comparison with the Galactic center. GAMMA-400 will be able to specify the constrain area.

Other objects. Other potentially interesting objects are other galaxies and their clusters, where dark matter is present and can emit gamma rays. GAMMA-400 with the highest energy resolution of $\sim 1 \%$ will have unique sensitivity for detecting dark matter.

\section{Acknowedgements}

This work was supported by the Space Council of Russian Academy of Sciences and the Russian Federal Space Agency

\section{References}

[1] F. Acero, et al., ApJS, 218, 23 (2015)

[2] O. Adriani et al. arXiv:1806.09728 (2018).

[3] A. Galper, et al., ASR, 51, 297 (2013)

[4] N. Topchiev, et al., J. of Phys.: Conf. Ser., 675 (2016) 032009

[5] A. Galper et al. Phys. Atomic Nucl., 80, 1141 (2017)

[6] A. Leonov et al. ASR, 56, 1538 (2015)

[7] G. Bertone. Particle dark matter - observations, models and searches. Cambridge Univ. Press, 2010

[8] K. Abazajian and M. Kaplinghat. Phys. Rev. D86 (2012) 083511, [arXiv:1207.6047]

[9] R. Bartels, S. Krishnamurthy, and C. Weniger. arXiv:1506.05104 (2015) 